\title{
Frequency-Shift a way to Reduce Aliasing in the Complex Cepstrum
}

\section{Bysted, Tommy Kristensen}

Published in:

Electronics, Circuits and Systems, 1998 IEEE International Conference on

Link to article, DOI:

10.1109/ICECS.1998.814091

Publication date:

1998

Document Version

Publisher's PDF, also known as Version of record

Link back to DTU Orbit

Citation (APA):

Bysted, T. K. (1998). Frequency-Shift a way to Reduce Aliasing in the Complex Cepstrum. In Electronics, Circuits and Systems, 1998 IEEE International Conference on (Vol. 3, pp. 557-560). IEEE. https://doi.org/10.1109/ICECS.1998.814091

\section{General rights}

Copyright and moral rights for the publications made accessible in the public portal are retained by the authors and/or other copyright owners and it is a condition of accessing publications that users recognise and abide by the legal requirements associated with these rights.

- Users may download and print one copy of any publication from the public portal for the purpose of private study or research.

- You may not further distribute the material or use it for any profit-making activity or commercial gain

- You may freely distribute the URL identifying the publication in the public portal 


\title{
Frequency-shift a way to reduce aliasing in the complex cepstrum
}

\author{
T.K. Bysted \\ Department of Information Technology \\ Technical University of Denmark \\ DK-2800 Lyngby, Denmark \\ Tel: +45 45253652 Fax: +4545880117 \\ E-mail: tk@it.dtu.dk
}

\begin{abstract}
Abstact
The well-known relation between a time signal and its frequency-shifted spectrum is in this paper introduced as an excellent tool for reduction of aliasing in the complex cepstrum. Using $N$ points DFTs the frequency-shift property will, used in the right way, reduce the aliasing error to a size which on average is identical to the one normally requiring $2 N$ points DFTs. The cost is an insignificant increase in the number of operations compared to the total number needed for the transformation to the complex cepstrum domain.
\end{abstract}

\section{Introduction}

The complex cepstrum is a versatile tool used in signal processing for different areas such as speech processing, machine diagnostic, marine seismology, deconvolution etc. The main reason for this is the ability to transform the convolution operator in the time domain into an addition operator in the complex cepstrum domain.

Although the transformation from a theoretical point is straightforward, two problems arise in practice [3][4]. The first one is the need for phase unwrapping which can be difficult especially if zeros or poles are located close to the unit circle. This problem has been addressed in a number of different papers resulting in some robust phase unwrapping algorithms. The second problem is aliasing which is the result of replacing the Fourier transform by the discrete Fourier transform (DFT), forcing the always infinite complex cepstrum into a periodic one. Although this problem has been known since the introduction of the complex cepstrum, no serious attempts have been made to reduce it without increasing the length of the involving DFTs.

In this paper a new transformation procedure called the frequency-shift (FST) method is proposed. The FST method computes the complex cepstrum using a different frequency grid of the DFTs giving an error which normally requires the double length of the involving DFTs. The reduction is obtained by using a frequency-shift relation between a complex cep- strum and its frequency-shifted Fourier transformed. This relation is introduced in section 2 as an extension to the normal relation known between a time signal and its frequency-shifted spectrum. In section 3 it is shown that the normally occurring aliasing in complex cepstrums is highly dependent on the introduced frequency-shift. This is utilised in section 4 to reduce or actually move some of the aliasing components from the real to the imaginary part of the complex cepstrum. An example is given in section 5 while section 6 is the conclusion.

\section{Frequency-shift}

From the basic signal processing theory it is well known that the frequency-shifting property defined as:

$$
e^{j \omega_{0} n} x(n) \stackrel{\mathcal{F}}{\longleftrightarrow} X\left(e^{j\left(\omega-\omega_{0}\right)}\right)
$$

can be used to change the zero location of the frequency axis. It is widely used in communication systems for modulation of signals. The property can also be used to change the normal frequency grid $\left(\omega_{k}=\frac{2 \pi}{N} k\right.$ where $\left.k \in[0, N-1]\right)$ of the DFT.

In this section the frequency-shifting property (1) is introduced in computation of the complex cepstrum. All transformations to the complex cepstrum domain are based on the continuous Fourier transform. The DFT case is considered in the next section.

If $X\left(e^{j\left(\omega-\omega_{0}\right)}\right)$ is obtained using $(1)$ and $x(n)$ is real, then it is well-known that the real and imaginary part of $X\left(e^{j\left(\omega-\omega_{0}\right)}\right)$ is symmetrical and antisymmetrical round $\omega=\omega_{0}$. The logarithm transform this frequency-shifted spectrum to the Fourier transformed of the complex cepstrum:

$$
\hat{X}\left(e^{j\left(\omega-\omega_{0}\right)}\right)=\ln \left(X\left(e^{j\left(\omega-\omega_{0}\right)}\right)\right)
$$

which is frequency-shifted too. Unwrapping the imaginary part of $\hat{H}\left(e^{j\left(\omega-\omega_{0}\right)}\right)$ and assuming continuity in $\omega= \pm \pi+\omega_{0}$, the real and imaginary parts are now continuous symmetrical and antisymmetrical round 
$\omega_{0} . \hat{X}\left(e^{j\left(\omega-\omega_{0}\right)}\right)$ can therefore be transformed to the complex cepstrum domain giving the relation:

$$
e^{j \omega_{0} n} \hat{x}(n) \stackrel{\mathcal{F}}{\longleftrightarrow} \hat{X}\left(e^{j\left(\omega-\omega_{0}\right)}\right)
$$

which shows that the frequency-shift property can also be used in connection with the complex cepstrum.

This general property is now used on signals which can be described by the ARMA model and the resulting frequency response is:

$$
\begin{aligned}
& H\left(e^{j\left(\omega-\omega_{0}\right)}\right)=|A| e^{-j\left(\omega-\omega_{0}\right) M_{0}} \\
& \frac{\prod_{i=1}^{M_{i}}\left(1-a_{i} e^{-j\left(\omega-\omega_{0}\right)}\right) \prod_{i=1}^{M_{0}}\left(1-b_{i}^{-1} e^{j\left(\omega-\omega_{0}\right)}\right)}{\prod_{i=1}^{P_{i}}\left(1-c_{i} e^{-j\left(\omega-\omega_{0}\right)}\right)}
\end{aligned}
$$

where $\left|a_{i}\right|$ and $\left|c_{i}\right|$ are smaller than one and $\left|b_{i}\right|$ is larger than one.

A direct transformation of (4) to the complex cepstrum domain is not possible due to the discontinuity of the phase at $\omega= \pm \pi+\omega_{0}$. However the continuity can be ensured by removing the linear-phase term $\left(-\left(\omega-\omega_{0}\right) M_{o}\right)[3][4]$.

The imaginary part of $\hat{H}\left(e^{j\left(\omega-\omega_{0}\right)}\right)$ is now guaranteed to be continuous and antisymmetrical. As a consequence $\hat{H}\left(e^{j\left(\omega-\omega_{0}\right)}\right)$ can be transformed to the complex cepstrum domain. Using (3) and the theory from [4] the complex cepstrum of (4) can be expressed as:

$$
\hat{h}_{\mathrm{s}}(n)= \begin{cases}-\sum_{i=1}^{M_{i}} \frac{a_{i}^{n} e^{j \omega_{o} n}}{n}+\sum_{i=1}^{P_{i}} \frac{c_{i}^{n} e^{j \omega_{o} n}}{n} & n>0 \\ \ln |A| & n=0 \\ \sum_{i=1}^{M_{o}} \frac{b_{i}^{n} e^{j \omega_{o} n}}{n} & n<0\end{cases}
$$

where the index $s$ indicates frequency-shift. From this equation the relation:

$$
\hat{h}(n)=e^{-j \omega_{0} n} \hat{h}_{\mathrm{s}}(n)
$$

between $\hat{h}_{\mathrm{s}}(n)$ and the true complex cepstrum $\hat{h}(n)$ is obvious.

In this section it has been shown that the frequency-shifting property can be used in connection with the complex cepstrum. The relation between the complex cepstrum domain and the Fourier transformed of the complex cepstrum is identical to the one existing between the time- and frequency domain.

\section{Aliasing}

After the theoretical case has been investigated this section is concentrated on the situation where the transformation to the complex cepstrum domain is based on DFTs.

In this case the complex cepstrum is a time-aliased version of the true complex cepstrum due to the forcing of the always infinite sequence into a periodical one [1][3][4]. The relation between the time-aliased and the true complex cepstrum $\left(\hat{h}_{\mathbf{p}}(n)\right.$ and $\left.\hat{h}(n)\right)$ is:

$$
\hat{h}_{\mathrm{p}}(n)=\sum_{r=-\infty}^{\infty} \hat{h}(n+r N)
$$

where $N$ is the length of the involving DFTs. Naturally, the same relation can also be written between the time-aliased and the true frequency-shifted complex cepstrum $\left(\hat{h}_{\mathrm{p}, \mathrm{s}}(n)\right.$ and $\left.\hat{h}_{\mathrm{s}}(n)\right)$.

Due to the use of DFTs, the time signal has to be zero outside the interval $n \in[0, N-1]$. This means it can be described by a MA (all-zero) model having the frequency response:

$$
\begin{aligned}
& H\left(e^{j \omega_{k}}\right)=|A| e^{-j \omega_{k} M_{\circ}} \\
& \quad \prod_{i=1}^{M_{i}}\left(1-a_{i} e^{-j \omega_{k}}\right) \prod_{i=1}^{M_{o}}\left(1-b_{i}^{-1} e^{j \omega_{k}}\right)
\end{aligned}
$$

where $M_{i}+M_{o}+1 \leq N$

Introducing frequency-shift in (8) (and removing the linear-phase component $\left.-\left(\omega-\omega_{0}\right) M_{0}\right)$ it can easily be shown that the time-aliased frequency-shifted complex cepstrum $\left(\hat{h}_{\mathrm{p}, \mathrm{s}}(n)\right)$ is:

$$
\begin{aligned}
& \hat{h}_{\mathrm{p}, \mathrm{s}}(n)= \\
& \begin{cases}-\sum_{i=1}^{M_{i}} \frac{a_{i}^{n} e^{j \omega_{0} n}}{n}+\delta_{\mathrm{s}, n>0} & n \in\left[1, \frac{N-1}{2}\right] \\
\ln |A|+\delta_{\mathrm{s}, n=0} & n=0 \\
\sum_{i=1}^{M_{o}} \frac{b_{i}^{n} e^{j \omega_{0} n}}{n}+\delta_{\mathrm{s}, n<0} & n \in-\left[\frac{N-1}{2}, 1\right]\end{cases}
\end{aligned}
$$

where

$$
\begin{aligned}
\delta_{\mathrm{s}, n>0}= & -\sum_{i=1}^{M_{i}} \sum_{r=1}^{\infty} \frac{a_{i}^{n+r N} e^{j \omega_{0}(n+r N)}}{n+r N}+ \\
& \sum_{i=1}^{M_{0}} \sum_{r=-\infty}^{-1} \frac{b_{i}^{n+r N} e^{j \omega_{0}(n+r N)}}{n+r N}
\end{aligned}
$$

Only $\delta_{s, n>0}$ is shown but the expressions for $\delta_{s, n=0}$ and $\delta_{\mathrm{s}, n<0}$ are similar.

Comparing this with the normal aliased complex cepstrum computed from $\hat{H}\left(e^{j \omega_{k}}\right)$ :

$$
\hat{h}_{\mathrm{p}}(n)= \begin{cases}-\sum_{i=1}^{M_{i}} \frac{a_{i}^{n}}{n}+\delta_{n>0} & n \in\left[1, \frac{N-1}{2}\right] \\ \ln |A|+\delta_{n=0} & n=0 \\ \sum_{i=1}^{M_{0}} \frac{b_{i}^{n}}{n}+\delta_{n<0} & n \in-\left[\frac{N-1}{2}, 1\right]\end{cases}
$$


where

$$
\delta_{n>0}=-\sum_{i=1}^{M_{i}} \sum_{r=1}^{\infty} \frac{a_{i}^{n+r N}}{n+r N}+\sum_{i=1}^{M_{o}} \sum_{r=-\infty}^{-1} \frac{b_{i}^{n+r N}}{n+r N}
$$

it is obvious that, due to aliasing, relation (6) is only true when $\omega_{0}= \pm p \cdot 2 \pi$ where $p \in 0,1,2, \ldots$; which is the same as no frequency-shift. This shows that the frequency-shift can be removed from the main cepstrum $(r=0)$ while this is not possible for the aliasing components $(r \neq 0)$. In the next section this dependence of the aliasing components upon the frequency-shift is utilised.

\section{Reducing aliasing}

So far it can be difficult to see what is gained by introducing this frequency-shift in computation of the complex cepstrum. But in this section the dependency of aliasing upon $\omega_{0}$ is investigated. It will be shown that for $\omega_{0} \neq \pm p \cdot \frac{\pi}{N}, p \in 0,1,2 \ldots$ aliasing is reduced leading to maximum reduction when $\omega_{0}= \pm(2 p-1) \cdot \frac{\pi}{2 N}, p \in 1,2,3 \ldots$

A time sequence $h(n)$ (having a frequency response described by (8)) is transformed to the complex cepstrum domain using the frequency-shifting property and equation (6). The resulting $\hat{h}_{\mathrm{p}}(n)=$ $e^{-j \omega_{0} n} \hat{h}_{\mathrm{p}, \mathrm{s}}(n)$ can be written as:

$$
\hat{h}_{\mathrm{p}}(n)= \begin{cases}-\sum_{i=1}^{M_{i}} \frac{a_{i}^{n}}{n}+\delta_{n>0} & n \in\left[1, \frac{N-1}{2}\right] \\ \ln |A|+\delta_{n=0} & n=0 \\ \sum_{i=1}^{M_{o}} \frac{b_{i}^{n}}{n}+\delta_{n<0} & n \in\left[-\frac{N-1}{2},-1\right]\end{cases}
$$

where

$$
\begin{aligned}
\delta_{n>0}= & -\sum_{i=1}^{M_{i}} \sum_{r=1}^{\infty} \frac{a_{i}^{n+r N} e^{j \omega_{0} r N}}{n+r N}+ \\
& \sum_{i=1}^{M_{0}} \sum_{r=-\infty}^{-1} \frac{b_{i}^{n+r N} e^{j \omega_{0} r N}}{n+r N}
\end{aligned}
$$

The contribution from the product $a_{i}^{n+r N} e^{j \omega_{0} r N}$ (or
$b_{i}^{n+r} e^{j \omega_{0} r N}$ ) to (14) can be split into four cases:

1. $\operatorname{Re}\left(a_{i}^{n+r N}\right) \wedge \operatorname{Re}\left(e^{j \omega_{0} r N}\right) \Rightarrow$ real

2. $\operatorname{Im}\left(a_{i}^{n+r N}\right) \wedge \operatorname{Re}\left(e^{j \omega_{0} r N}\right) \Rightarrow$ imaginary

3. $\operatorname{Re}\left(a_{i}^{n+r N}\right) \wedge \operatorname{Im}\left(e^{j \omega_{0} r N}\right) \Rightarrow$ imaginary

4. $\operatorname{Im}\left(a_{i}^{n+r N}\right) \wedge \operatorname{Im}\left(e^{j \omega_{0} r N}\right) \Rightarrow$ real

Having in mind that a complex cepstrum of a real sequence $h(n)$ is always real [4], it is evident that only real aliasing components (cases 1 and 4 ) can cause problems. In fact case 1 is the only problem because a component from case 4 will be cancelled by the complex conjugated zero $a_{i}^{*}\left(b_{i}^{*}\right)$. This shows that choosing $\omega_{0}$ in such a way that $e^{j \omega_{0} r N}$ is real $\forall r \neq 0$, then the complex cepstrum is maximum corrupted by aliasing, whereas a value making the product imaginary $\forall r \neq 0$ means no aliasing. Unfortunately, the former is easily obtained $\left(\omega_{0}= \pm p \cdot \frac{\pi}{N}, p \in 0,1,2 \ldots\right)$ while the latter is impossible. Because a complete moving (of all aliasing components) to the imaginary part of the cepstrum is impossible, the best which can be done is to move as many components as possible. It can easily be verified that the optimal value for this is $\omega_{0}= \pm(2 p-1) \cdot \frac{\pi}{2 N}, p \in 1,2,3, \ldots$, where all components for $r$ odd is moved.

Using $\omega_{0}=\frac{\pi}{2 N}$ the real part of $(14)$ can be written as:

$$
\begin{aligned}
\operatorname{Re}\left(\delta_{n>0}\right)= & -\sum_{i=1}^{M_{i}} \sum_{r=1}^{\infty} \frac{a_{i}^{n+r 2 N}(-1)^{r}}{n+r 2 N}+ \\
& \sum_{i=1}^{M_{0}} \sum_{r=-\infty}^{-1} \frac{b_{i}^{n+r 2 N}(-1)^{r}}{n+r 2 N}
\end{aligned}
$$

resulting in the following equation describing timealiasing in the complex cepstrum using the FST method (frequency-shift method where $\omega_{0}=\frac{\pi}{2 N}$ ) and $\mathrm{N}$ points DFTs:

$$
\hat{h}_{\mathrm{p}}(n)=\sum_{r=-\infty}^{\infty}(-1)^{r} \hat{h}(n+r 2 N)
$$

where $n \in\left[-\frac{N-1}{2}, \frac{N-1}{2}\right]$.

A comparison between (16) and (7) shows that the average size of the aliasing error using FST is identical to the one normally requiring $2 N$ length DFTs.

This section has proofed that aliasing (in the complex cepstrum) is sensitive not only to the transformation length $N$ but also to the choice of sampling points in the frequency domain. The FST method change these from $\omega_{k}=\frac{2 \pi}{N} k$ (normal grid of the DFT) to $\omega_{k}=\frac{2 \pi}{N} k-\frac{\pi}{2 N}$ and in that way aliasing is reduced considerably.

\section{Example}

This section is used to illustrate the ability of th FST method for reduction of aliasing.

An equiripple 121 coefficient lowpass linear-pha FIR-filter designed using the Remez algorithm a. the specifications:

$$
G(f)= \begin{cases}1 & 0 \leq f \leq 0.25 \\ 0 & 0.3 \leq f \leq 0.5\end{cases}
$$



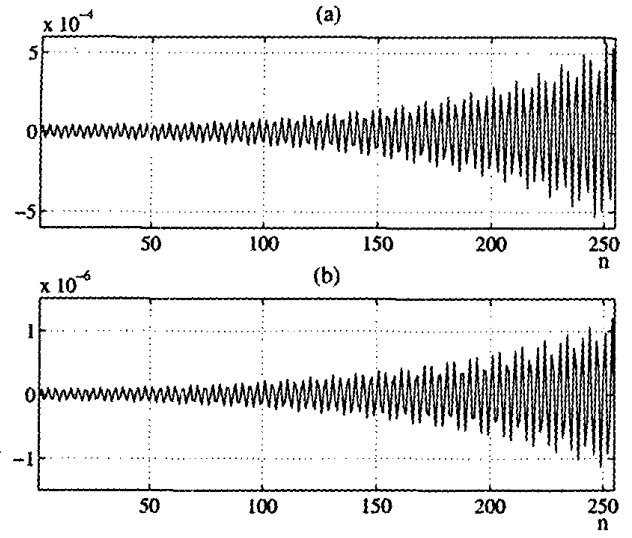

Figure 1: Aliasing for traditional method (a) $N=$ 512 and (b) $N=1024$ points DFTs
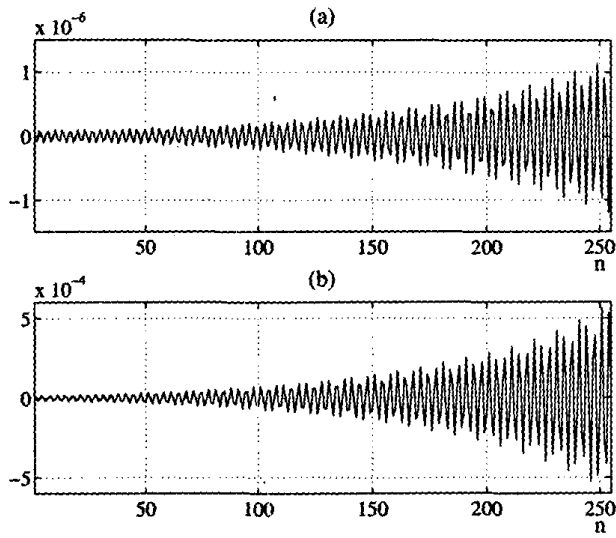

Figure 2: Aliasing (a) and imaginary part of complex cepstrum (b) when FST method and $N=512$ points DFTs is used.

is transformed to the complex cepstrum domain.

Transformation of linear-phase FIR-filters to the complex cepstrum domain is often done due to the ability to separate zeros out- and inside the unit circle (see equation (5)). Due to this the complex cepstrum is an excellent tool in transformation from linear- to minimum-phase FIR-filters [2][5].

The impulse response $h(n)$ is transformed to the omplex cepstrum domain using the conventional transormation method ( $N=512$ and $N=1024)$ and he proposed FST method $(N=512)$. Figures 1 B) and (b) show the aliasing error using the normal ethod while the error using the FST is shown in gure 2 (a). Only the causal part ( $n$ positive) is own, but because $h(n)$ is a linear-phase FIR-filter : noncausal part is identical. From these figures $s$ obvious that the proposed method gives an erwhich normally requires the double length of the rs.
To illustrate the moving of the error from the real to the imaginary part of the complex cepstrum, Figure 2 (b) depicts the imaginary part which can be described by the expression:

$$
\begin{gathered}
\operatorname{Im}\left(\hat{h}_{\mathrm{p}}(n)\right)=\sum_{r=1}^{\infty}\left(i ( - 1 ) ^ { r } \left(\sum_{i=1}^{M_{i}} \frac{a_{i}^{n+(2 r-1) N}}{n+(2 r-1) N}\right.\right. \\
\left.\left.+\sum_{i=1}^{M_{0}} \frac{b_{i}^{n-(2 r-1) N)}}{n-(2 r-1) N}\right)\right) n \in\left[1, \frac{N-1}{2}\right]
\end{gathered}
$$

This shows that the imaginary part is the difference between the aliasing caused by zeros inside and zeros outside the unit circle. When $n$ is in the vicinity of zero, these two contributions are nearly identical but as $n \rightarrow \frac{N-1}{2}$ the zeros outside will dominate [1] and the amplitude of Figure 2 (b) will go towards the amplitude of Figure 1 (a).

\section{Conclusion}

The frequency-shifting property has been introduced in the area of complex cepstrum analysis. In the theoretical case the relation between the complex cepstrum and its Fourier transformed is identical to the frequency-shift property relating the time and frequency domain. In practice where the complex cepstrum is aliased, the property is only true for the main cepstrum components and not for the aliased components. This is utilized in a proposed transformation method called frequency-shift (FST) method to reduce aliasing.

Using the FST method and $N$ points DFTs the average size of the aliasing is identical to one normally requiring $2 N$ points DFTs. The cost of this considerable reduction is a small rise in the number of operations compared to a conventional transformation to the complex cepstrum domain.

\section{References}

[1] T.K. Bysted, "Aliasing in the Complex Cepstrum of Linear-Phase Signals", Proc. ICICS,pp. 1598-1602, 1997.

[2] T.K. Bysted, K.J. Jensen and H. Gaunholt, "Design of Linear- and Minimum- phase FIR-equalizers", Proc. BEC-96,pp. 401-404, 1996.

[3] D.G. Childers, D.P. Skinner and R.C. Kemerait, "The Cepstrum: A guide to processing", Proc. IEEE, vol. 65, pp. 1428-1443, 1977.

[4] A.V. Oppenheim and R.W. Schafer, "Discrete-time signal processing", Prentice Hall, 1989.

[5] G.R. Reddy, "Design of Minimum-Phase FIR digital filter through Cepstrum", Electron. Lett., vol. 22, pp. 1225-1227, 1986.

[6] P.L. Stoffa, P. Buhl and G.M. Bryan, "Cepstrum aliasing and the calculation of the Hilbert transform", Geophysics, vol. 39, pp. 543-544, 1974. 